\title{
Coal Conveying Fault Diagnosis Design of Coal mining machine Based On Software Control
}

\author{
GAO Jingyuan ${ }^{1, a}$ \\ ${ }^{1}$ Hebei Vocational \&Technical College of Building Materials QinHuangDaoHeBei 066000,China \\ a27125412@qq.com
}

Keywords: coal mining machine; coal conveying system; neural network; fault diagnosis; genetic algorithm and particle swarm algorithm (GA-PSO)

\begin{abstract}
The coal conveying system is the most important composition unit of continuous mining machine, the performance of coal conveying system plays a decisive role in production efficiency, this equipment is easy to cause the fault, in this paper, the faults often appeared in this equipment and the fault reasons are analyzed, genetic algorithm (GA) and particle swarm optimization (PSO) are taken as the optimization tool, and then combined with the method of BP neural network, the fault diagnosis of coal conveying system is researched. The analysis results show that, this method can make the BP neural network away from local minima effectively, the accuracy of the equipment is increased, and then the fault diagnosis of equipment can be carried out quickly, conveniently and accurately.
\end{abstract}

\section{Introduction}

Continuous coal mining machine is a combined unit with multiple functions, it is mostly applied in short wall mining and driving openings, it is widely applied in the coal mine and other mines mining, it has good application value and application prospect. The most important composition unit of continuous mining machine is the coal conveying system, the performance of coal conveying system plays a decisive role in production efficiency, this equipment is easy to cause the fault, so the convenient fault diagnosis method is very meaningful for improving the coal mining efficiency. The traditional fault diagnosis methods use the BP neural network method, and it has a number of weaknesses, in order to improve the accuracy of fault diagnosis, BP neural network is optimized, the most effective optimization method is GA-PSO hybrid algorithm, this algorithm has many advantages, there are significant advantages in terms of weight training and convergence speed, and it can effectively avoid the local minimum value. Therefore, in this paper, the fault diagnosis of coal conveying system uses the method of GA-PSO hybrid BP neural network for optimization.

\section{Fault analysis and detection method}

A coal transport system is driven by the hydraulic system, it is comprised with hydraulic lifting cylinder, swing hydraulic cylinder, the scraper chain and scraper and the unloading roller, the system composition structure is shown as Figure 1.

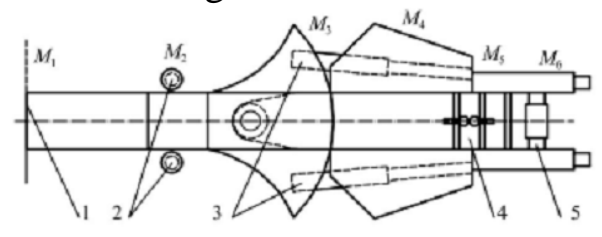

Figure 1 Coal conveying system structure of the coal mining system

\section{A. Fault phenomenon and fault reasons}

Coal transport system will have the following some troubles: $X_{1}$ scraper chain has the low transmission speed, $X_{2}$ - scraper chain has a transmission rate of the instability, $X_{3}$ - unstable 
transmission of scraper chain causes noise and unstable vibration. $X_{4}$ - operating temperature of bottom shaft 1 is too high. $X_{5}$ - operating temperature of unloading roller 5 is too high, $X_{6}$ - scraper chain 4 tightness has problems, the system cannot move freely in the vertical direction, $X_{8}$ - the system cannot move freely in horizontal direction. $X_{9}$ - the circulating pipeline temperature of the system is too high, $X_{10}$ - the circulation pipeline has leakage problem.

After the analysis of the fault phenomena, the fault reasons can be expressed as follows: $Y_{1}$ - the system of clutch slipping; $Y_{2}$ - bottom shaft and sprocket of the system are damaged due to friction; $Y_{3}$ - lack of lubricating oil in the bearing parts; $Y_{4}$ - the system for bearing unloading lack of lubrication; $Y_{5}$ - hydraulic cylinder with lifting effect appears the oil spill; $Y_{6}$ - hydraulic cylinder with oscillating action appears the oil spill; $Y_{7}$ - circulating pipeline of the system is damaged; $Y_{8}$ damage occurred in hydraulic cylinder control valve has the lifting function; $Y_{9}$ - the system cannot run in the latter part; $Y_{10}$ - the system cannot operate in normal in the tension position. $Y_{11}$ - sealing effect of circulation pipeline is failure. $Y_{12}$ - due to impurity, hydraulic pressure fluid is not pure. $Y_{13}$ - volume of hydraulic pressure fluid is insufficient. $Y_{14}$ - hydraulic cylinder control valve with oscillating action is damaged.

\section{B.Detection method}

In the coal conveying system, $M_{1}-M_{66}$ information detection points are set, in each detection points, the sensors are installed, corresponding to each key point, signal are collected, it is shown in Figure 1. For the detection of fault signals $X_{2}, X_{3}$, and ${ }^{X_{4}}$, the temperature sensor $M_{1}$ is installed in the system of bearing seat, temperature sensor $M_{2}$ is installed in the pipeline of the hydraulic system of lifting the cylinders for detecting the fault signals $X_{7}$ and ${ }^{X_{10}}$. Pressure sensor ${ }^{M_{3}}$ is installed in the pipeline of the hydraulic cylinder swing of the system for detecting the fault signals $X_{8}$ and ${ }^{X_{10}}$. Pressure sensor ${ }^{M_{4}}$ is installed in the in the central of the system for detecting the fault signals $X_{1}, X_{3}$ and $X_{6}$, Pressure sensor $M_{6}$ is installed in the in the roller bearing seat of the system for detecting the fault signals $X_{2}, X_{3}$ and $X_{5}$.

\section{Mathematical model of fault diagnosis neural network}

The mathematical model is consisted of three layers of neural network. The three layer neural network has 14 neurons output layers and hidden layer with 10 neurons, and input layer with 10 neurons. The net input value from the input layer to the hidden layer can be calculated by formula:

$$
n_{1 j}=\sum_{i=1}^{10} \omega_{i j} X_{i}-\theta_{1 j} \quad(\mathrm{j}=1,2, \ldots, 10)(1)
$$

Where, $\theta_{1 j}, \omega_{i j}$ are weights and threshold from the input layer to the output layer, $X_{i}$ is the input fault values of the sample, net input value from the hidden layer to the output layer can be calculated by formula:

$$
n_{2 k}=\sum_{j=1}^{10} \frac{v_{j k}}{1+\mathrm{e}^{-\lambda n 1 j}}-\theta_{2 k} \quad(\mathrm{k}=1,2, \ldots, 14)(2)
$$

Where, $\theta_{2 k}$ and $v_{j k}$ are weights and threshold from the hidden layer to the output layer. $\lambda$ is the speed coefficient, the value is 1 . Output error of neural network can be calculated by the formula (3):

$$
E=\frac{1}{2} \sum_{k=1}^{14}\left(Y_{k}-\frac{1}{1+\mathrm{e}^{-\lambda n 2 k}}\right)^{2}
$$


In the formula, $Y_{k}$ is the output value of the sample.

Finally, the gradient algorithm is used to train the thresholds $\theta_{2 k}, \theta_{1 j}$, and the weights $v_{j k}$ and $\omega_{i j}$, the expert system knowledge base can be obtained.

\section{BP network optimization based on GA and PSO hybrid algorithm and the simulation analysis}

\section{A.Hybrid optimization of GA and PSO and the characteristics of BP network}

Genetic algorithm and particle swarm algorithm are combined with serial and parallel calculation, it can maintain rapid convergence in the limited improvement of precision, the particle swarm algorithm is proposed. Because the two algorithms are fused, the advantages and features of the two algorithms are outstanding, it can optimize the BP neural network, and the advantages are effective.

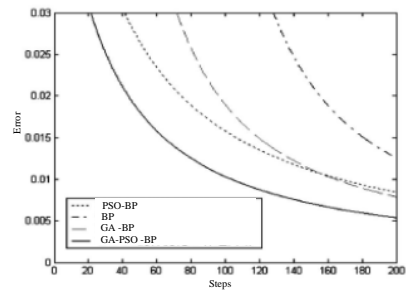

Figure 2. Error curve

Firstly, the operator operation of particle swarm is taken, in which the population number $\mathrm{m}=60$, for each species $=\left\{\omega_{i j}, \theta_{1 j}, v_{j k}, \theta_{2 k}\right\}$. Fitness value can be combined with $\mathrm{m} \times \mathrm{n}$ and formula (3), fitness value is obtained, update speed $N_{i+1}=c_{0} N_{i}+c_{1}\left[P_{i}-x_{i}\right]+c_{2}\left[G_{i}-x_{i}\right](i=1,2 \cdots m)$, the position $x_{i+1}=x_{i}+N_{i+1}$ can be obtained. The weighted coefficient $c_{0}=1$, learning factors $c_{1}$ and $c_{2}$ are equivalent to 2 . To meet the requirement of accuracy, the genetic operators are taken, using real number coding, crossover rate is 0.95 , the mutation rate and fitness are related. In this process, when the maximum generation is achieved, then jumps back into the PSO operator, until meet the total accuracy after the end of the program, otherwise taken loop calculation. The initial value of the BP neural network is set as the thresholds $\theta_{1 j}, \theta_{2 k}$ and weights $\omega_{i j}, v_{j k}$, after learning and training, the knowledge library of expert system also can be formed.

\section{B.Simulation results an analysis}

The traditional BP neural network algorithm, optimized BP neural network, optimized BP neural network based on GA, and hybrid GA-PSO optimized BP neural network are taken in the simulation for comparison, the MATLAB simulation software are used, and the error curve is obtained, which is shown in Figure 2.

The simulation results show that, the BP neural network optimized by GA has better performance than the BP neural network optimized by PSO, convergence precision is higher, but the convergence time is longer. However, the traditional BP neural network has no advantage in convergence time and accuracy, the improved algorithm can overcome the inherent shortcomings of BP neural network, which can optimize the BP neural network, the performance is more effective.

However, the traditional BP neural network has no advantage in the convergence time and convergence accuracy, the convergence precision of BP neural network algorithm based on GA-PSO hybrid optimization has very obvious advantages. The error comparison results of several optimization methods are expressed in Table 1. For the A-PSO hybrid optimization BP neural network algorithm, maximum error and the minimum error was the smallest. Thus, the BP neural network of GA-PSO hybrid optimization has the advantages of high precision, it has fast convergence. 
Table 1 Comparison of errors

\begin{tabular}{ccccc}
\hline $\begin{array}{c}\text { Network } \\
\text { types }\end{array}$ & $\begin{array}{c}\text { Traditional BP } \\
\text { neural network }\end{array}$ & $\begin{array}{c}\text { BP neural network with } \\
\text { GA optimization }\end{array}$ & $\begin{array}{c}\text { BP neural network PSO } \\
\text { optimization }\end{array}$ & $\begin{array}{c}\text { BP neural network } \\
\text { GA-PSO }\end{array}$ \\
\hline $\begin{array}{c}\text { Average } \\
\text { error }\end{array}$ & 0.01349 & 0.0078954 & 0.0084649 & 0.0053737 \\
$\begin{array}{c}\text { Minimum } \\
\text { error }\end{array}$ & 0.01081 & 0.004923 & 0.0048315 & 0.0043311 \\
$\begin{array}{c}\text { Maximum } \\
\text { error }\end{array}$ & 0.01983 & 0.015021 & 0.015335 & 0.0077805 \\
\hline
\end{tabular}

\section{BP neural network expert system with GA-PSO}

First of all, SQL2000 is taken as the knowledge base to store the fault history table, knowledge base tables and sample learning table. $\mathrm{VC}++6.0$ is taken as programming platform, friendly interactive interface is obtained, combined with the internal embedded with powerful image function of MATLAB, the continuous mining machine conveying fault diagnosis system is completed based on GA and PSO hybrid BP neural network optimization. The user can select the user level in the expert system, and it is shown in Figure 3. The fault diagnosis results of coal transport system of continuous mining machine are shown in Figure 4.

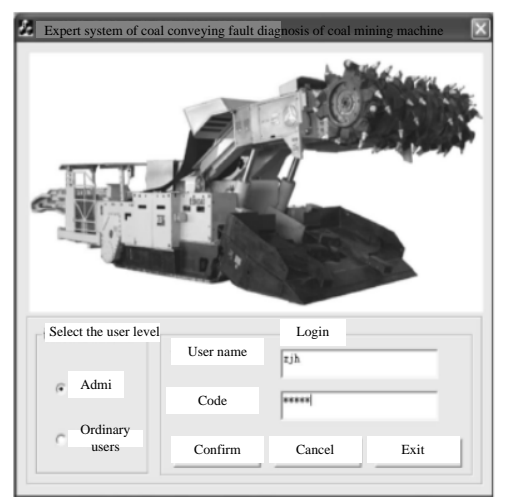

Figure 3. Main interface of expert system

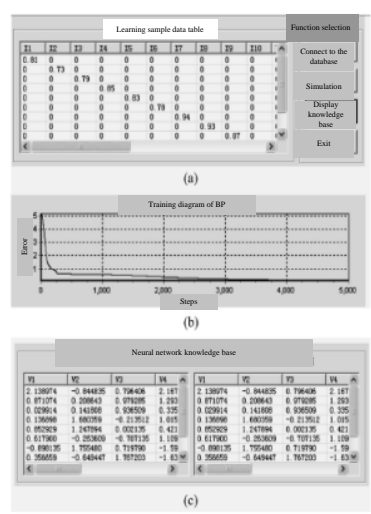

Figure 4. Interface and fault diagnosis results

\section{Conclusions}

(1) In this paper, the BP neural network is optimized based on genetic algorithm (GA) and particle swarm optimization algorithm (PSO), the fault diagnosis of coal conveying system is accurate and rapid.

(2) This method is used on continuous shearer coal fault diagnosis, it not only makes the system performance is more stable, but also can greatly improve the production efficiency of the system.

\section{References}

[1] YANG Ming, HAO Liang, XU Dianguo. Online suppression of mechanical resonance based on adapting notch filter[J]. Journal of Harbin Institute of Technology, 2014,46(4):63-69.

[2] WANG Rui, MA Yan. DOA Estimation of Wideband Linear Frequency Modulated Pulse Signals Based on Fractional Fourier Transform[J]. Acta Armamentarii, 2014,35(3):421-427.

[3] Ou Shi-feng, Gao Ying, Zhao Xiao-hui. Adaptive Combination Algorithm and Its Modified Scheme for Blind Source Separation[J]. Journal of Electronics \& Information Technology, 2011, 33(5): 1243-1247.

[4] PENG Li-ying. Traffic Data Anti Step Fusion Algorithm Based on Improved Sliding Mode Disturbance Control Rule[J]. Control Engineering of China, 2014,21(4): 515-519.

[5] Liu Xiangdong. Data Clustering Algorithm and Software Design Based on Disturbance Searching of Logistic Series[J]. Bulletin of Science and Technology, 2014,30(2): 161-163.

[6] ZHANG Ren-shang. Network Intrusion Detection System Based on Expert System and Neural Network[J]. Computer Simulation, 2012, 29(9): 162-165. 